\title{
Profiling and Tracing Support for Java Applications
}

\section{Document Version}

Accepted author manuscript

Link to publication record in Manchester Research Explorer

\section{Citation for published version (APA):}

Nisbet, A., Nobre, N. M., Riley, G., \& Luján, M. (2019). Profiling and Tracing Support for Java Applications. 1. Paper presented at 10th ACM/SPEC International Conference on Performance Engineering (ICPE 2019), Mumbai, India.

\section{Citing this paper}

Please note that where the full-text provided on Manchester Research Explorer is the Author Accepted Manuscript or Proof version this may differ from the final Published version. If citing, it is advised that you check and use the publisher's definitive version.

\section{General rights}

Copyright and moral rights for the publications made accessible in the Research Explorer are retained by the authors and/or other copyright owners and it is a condition of accessing publications that users recognise and abide by the legal requirements associated with these rights.

\section{Takedown policy}

If you believe that this document breaches copyright please refer to the University of Manchester's Takedown Procedures [http://man.ac.uk/04Y6Bo] or contact uml.scholarlycommunications@manchester.ac.uk providing relevant details, so we can investigate your claim.

\section{OPEN ACCESS}




\title{
Profiling and Tracing Support for Java Applications
}

\author{
Andy Nisbet, Nuno Miguel Nobre, Graham Riley, and Mikel Luján \\ School of Computer Science, University of Manchester, Manchester, UK \\ \{name.surname\}@manchester.ac.uk
}

\begin{abstract}
We demonstrate the feasibility of undertaking performance evaluations for JVMs using: (1) a hybrid JVM/OS tool, such as async-profiler, (2) OS centric profiling and tracing tools based on Linux perf, and (3) the Extended Berkeley Packet Filter Tracing (eBPF) framework where we demonstrate the rationale behind the standard of fwaketime tool, for analysing the causes of blocking latencies, and our own eBPF-based tool bcc-java, that relates changes in microarchitecture performance counter values to the execution of individual JVM and application threads at low overhead.

The relative execution time overheads of the performance tools are illustrated for the DaCapo-bach-9.12 benchmarks with OpenJDK9 on an Intel Xeon E5-2690, running Ubuntu 16.04. Whereas sampling based tools can have up to $25 \%$ slowdown using $4 \mathrm{kHz}$ frequency, our tool bcc-java has a geometric mean of less than $5 \%$. Only for the avrora benchmark, bcc-java has a significant overhead (37\%) due to an unusually high number of futex system calls. Finally, we provide a discussion on the recommended approaches to solve specific performance use-case scenarios.
\end{abstract}

\section{CCS CONCEPTS}

- General and reference $\rightarrow$ Performance; • Software and its engineering $\rightarrow$ Virtual machines.

\section{ACM Reference Format:}

Andy Nisbet, Nuno Miguel Nobre, Graham Riley, and Mikel Luján. 2019. Profiling and Tracing Support for Java Applications. In Tenth ACM/SPEC International Conference on Performance Engineering (ICPE '19), April 7-11, 2019, Mumbai, India. ACM, New York, NY, USA, 8 pages. https://doi.org/10. $1145 / 3297663.3309677$

\section{INTRODUCTION}

We briefly survey standard performance evaluation approaches and tools, and their limitations for Java Virtual Machines (JVMs) concerning Garbage Collection (GC) log files, heap analysis, lock contention, processor core sampling, and bytecode based instrumentation for the measurement of application specific performance metrics (Table 1 summarises tool features and capabilities). Logging involves turning on JVM flags to monitor specific JVM subsystem behavior (e.g. JIT compilation or GC). The two main approaches to profiling are tracing and sampling. Tracing instruments code to measure microarchitecture or system performance metrics. Sampling

We acknowledge support of the EU Horizon 2020 ACTiCLOUD 732366, and the UK EPSRC PAMELA EP/K008730/1 project grants.

ICPE '19, April 7-11, 2019, Mumbai, India

(C) 2019 Copyright held by the owner/author(s). Publication rights licensed to ACM.

This is the author's version of the work. It is posted here for your personal use. Not for redistribution. The definitive Version of Record was published in Tenth ACM/SPEC International Conference on Performance Engineering (ICPE '19), April 7-11, 2019, Mumbai, India, https://doi.org/10.1145/3297663.3309677. profilers repeatedly collect the stack trace of called functions/methods, that describe the code running on the processing core, on-core, at the sampled moments in time.

The Unix OS perf tool is becoming widely used as it supports both sampling and tracing. In the Java community, sampling based profiling is widely implemented with $\mathcal{F V M}$ Tools Interface (JVMTI) agent support using GetCallTrace (e.g. JProfiler). This approach is reliant on restricting sampling to safe-points, that are inserted by the JIT compiler to support GC. Such restrictions generate bias, and can lead to incorrect performance information. DTrace was a key tool popularizing tracing on Solaris, and then ported to FreeBSD, NetBSD, Mac OS, and in 2018 to Windows. Since 2016, Linux integrated similar functionality via the eBPF framework (Kernel version 4.9). This paper demonstrate the feasibility of undertaking performance evaluations for unmodified JVMs using either, (1), a hybrid JVM/OS tool, such as async-profiler, (2), OS-centric profiling and tracing tools based on perf, or (3) our own eBPF-based tracing tool, bcc-java. The contributions of this paper are:

- We measure the overheads of on-core sampling with profiling for $100 \mathrm{~Hz}$ ( 1 sample every $10 \mathrm{~ms}$ ), $1 \mathrm{kHz}$ (every $1 \mathrm{~ms}$ ) and $4 \mathrm{kHz}$ (every $250 \mu \mathrm{s}$ ) for the DaCapo benchmarks. We find that $1 \mathrm{kHz}$ sampling can be used with the async-profiler and perf/perf-map-agent with a reasonable geomean overhead of less than $5.2 \%$ and $11.2 \%$ respectively compared to normal execution.

- We show how flamegraphs produced using perf combined with perf-map-agent can be used to identify where JIT based compilation has failed to inline successfully.

- We report that heap allocation based profiling using the async-profiler is low overhead, at less than $2.8 \%$, for all benchmarks, and that flamegraphs enable developers to easily determine the main allocation sources in their programs.

- We present a low overhead tracing tool bcc-java (see Section 5) with a geometric mean overhead of $3.6 \%$ for characterizing the performance of all service and application threads created by a JVM. The bcc-java tool is developed on top of Linux eBPF Compiler Collection $(\mathrm{BCC})[12,23]$ for adding tracing support to operating system kernels and applications. Performance counter measurements such as instructions executed, processor cycles and cache misses can be directly related to application thread IDs, and to VM services. The monitoring techniques in the tool have generic applicability that could be deployed to characterize any multi-threaded application. - We demonstrate how the BCC offwaket ime tracing tool can be used to produce flamegraph visualizations that describe important aspects of thread blocking and wakeup execution behavior without requiring JVM modifications.

- We summarize the main features of the tools async-profiler, perf, offwaketime, and our new bcc-java tool in Table 1.

Section 2 generally discusses the capabilities of log file analysis tools targeting GC and JVM related memory performance analysis. Section 3 presents Flamegraphs [13] and how to interpret 
them for JVMs. Section 4 explains the capabilities of traditional Java based profiling tools and their limitations. The benefits of AsyncGetCallTrace based profiling, and the stack fragment sampling approach of [15] are discussed. Section 5 explains the rationale behind the design of the eBPF/BCC tools and the tracing tool that we have built. Section 6 presents our experimental methodology and performance overhead analysis. Section 7 presents guidelines for the use of performance evaluation tools under different use-cases, and finally Section 8 discusses our conclusions.

\section{RELATED WORK: GC \& JVM ANALYSIS}

JVM implementations support the logging of GC statistics by statically or dynamically turning on JVM flags. The statistics typically concern object allocations, usage of heap memory spaces, stopthe-world (STW) pauses, and total execution times for each GC invocation. STW pauses occur when all application threads are blocked during GC. Post-processing or runtime analysis of logs can be performed by tools such as [10, 9, 11, 20]. Lengauer et al. [17] presents an in-depth analysis of the GC behavior of Java benchmarks including DaCapo using G1 GC in the HotSpot JVM. Automated processing of logs can aid identification and solution of GC related performance problems, such as increasing the maximum heap size, changing the ratio of younger to older generation heap sizes, and changing heuristics for moving objects into different heap spaces. Dynamic dashboard visualizations are typically used to present information concerning the relative health and efficiency of applications in production environments.

However, GC log analysis cannot help in identifying or resolving which application code is the cause of high memory allocation and/or leaks. The JVM can provide a heap dump snapshot of the current live objects at relatively high cost. Such dumps may help developers to determine why GC execution and pause times might be higher than expected. Unfortunately heap dumps lack information on the allocation site of an object, nor can a dump help determine which thread allocated an object. Object deallocations can only be detected by comparing two subsequent dumps and finding that an object was removed. This is difficult as objects often change their heap storage location during GC where objects move between memory generation regions, and in heap compaction. Object allocation profiling and recording of every object using standard JVMs are typically based on expensive [24] combinations of bytecode based instrumentation, and stack traces. Consequently, many tools only record allocations above minimum sizes, and/or every $n$-th allocation in order to reduce overhead.

AntTracks [16] is a customized HotSpot based JVM that instruments object allocation, movement and deallocation with a low logging overhead of less than $4.68 \%$. The recorded events are sufficiently detailed to enable the heap's state to be reconstructed offline, for the beginning and end of every GC cycle by incrementally applying the effect of events described in trace files. Thread local allocation buffers (TLABs) are used to store event traces, with data compression to minimise trace collection and storage overheads.

Cao et al. [6] evaluated the power and performance requirements of managed runtime workload execution. Their work considered single-ISA heterogeneous asymmetric multicore processor (AMP) hardware with processor cores that are optimised for different power-performance tradeoffs. The main findings of interest are that GC accounts for $10 \%$ of processor execution cycles on average, and nearly $40 \%$ for lusearch from the DaCapo benchmarks.

Sartor et al. [21] presented techniques to analyze the scalability of managed language applications with speedup stacks. Lightweight OS kernel modules were used to monitor an application and its VM service threads scheduling behavior with an overhead of up to $1.15 \%$ but, typically under $1 \%$. They perform experiments on one node of an Intel Xeon E5-2650L server consisting of 2 sockets, each with $20 \mathrm{MB}$ shared LLC, 8 physical cores, with hyper threading enabled giving 16 logical cores on each socket, and a 64-bit 3.2.37 Linux kernel. Sufficient logical cores are present to ensure that the OS does not need to schedule out a thread other than for synchronization or I/O. A problem with this approach is that it is not expected to be portable across processor ISA architectures, and it may require recompilation for each new kernel release. For their experiments, they used JikesRVM [4] with the DaCapo lusearch, pmd, sunflow and xalan benchmarks. JikesRVM was modified to record the JVM application, compilation and garbage collection thread type process identifiers (PIDs) to enable their kernel modules to attribute microarchitectural performance counter measurements to specific JVM thread types. The speedup stack visualization helps developers to determine if optimization efforts that focus on application, or JVM runtime services are likely to improve performance. Our bcc-java tool development was motivated by [21].

Hofer et al. [14] modified OpenJDK8u45 to observe and trace events related to lock contention with a mean runtime overhead of $7.8 \%$. The call-stacks of a thread blocking on a lock, and the thread holding the lock causing the blocking were recorded. They also presented a tool for users to identify locking bottlenecks in their code by analysing the traced events. They identified that many call-stacks that use locks are actually identical, therefore it is only necessary to maintain a hash set of known call-stacks.

The BCC offwaketime tool performs a similar task to [14], except that it uses eBPF's OS level tracing of kernel events associated with thread scheduling to determine: (1) the call-stack of a blocked thread, (2) its blocked time duration, and (3) the call-stack of the waking thread, which causes the scheduling state of the blocked thread to change to runnable. The call-stack of the blocking thread does not change whilst it is off-core and blocked. Hence, its callstack need only be recorded when it meets some selection criteria, such as belonging to a process that we are interested in, and if its blocking duration is within a specified range. This enables efficient and selective tracing of call-stack blocking and wakeup behavior due to thread interactions.

\section{FLAMEGRAPH VISUALIZATIONS}

Brendan Gregg [13] has shown that scalable vector graphic (SVG) visualizations of flamegraphs, see Figure 1, can aid identification of performance critical code from sampled stack traces. Stack traces can be related to processing core time, or to the change in a quantity, such as last level cache misses, from a hardware counter. In this way it is possible to produce flamegraphs that characterize the microarchitectural behavior of an application's execution. SVGs enables flamegraphs to be viewed, zoomed in/out, and searched for text inside web browsers. The percentage contribution of a 


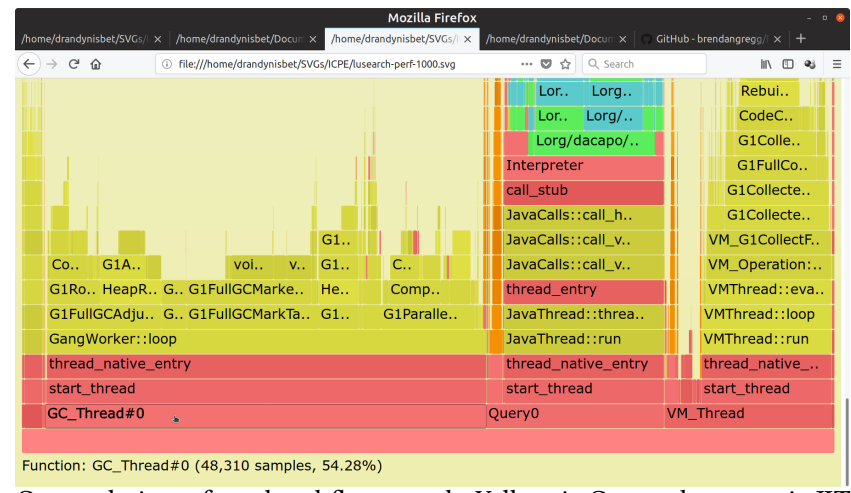

Cropped view of rendered flamegraph. Yellow is $\mathrm{C}++$ code, green is JIT compiled Java, red is native/library, and orange is kernel code. Constrained heap size to make GC significant.Inlined methods appear as teal/blue.

Figure 1: Flamegraph: lusearch processing of on-core stack traces using perf, perf-map-agent with $1000 \mathrm{~Hz}$ sampling.

method is easily determined by searching for its name, whereupon each instance of the method in the graph is highlighted and its overall contribution to the total number of samples is displayed at the bottom right hand corner. This can be especially important for determining the relative contribution of methods having many different call-sites in an application.

In a flamegraph, any stack traces having identical callers are merged, then any non-identical child callee nodes in the collected traces appear as a new control flow path. Control flow paths are visualized by presenting the unique names of methods inside rectangular blocks that are typically organized lexicographically in order from left to right. Divergence in a control flow path is indicated by more than one rectangular block being stacked on top of another, such as for the blocks stacked on top of GangWorker: : loop in our Figure 1. The topmost frame in a flamegraph is the method that was executing when a call-stack was sampled. The topmost methods will be identified correctly as long as any sampling skid ${ }^{1}$ [7] does not cause an incorrect method to be attributed. Note how the use of different colours can be used to distinguish between inlined Java, kernel, JIT compiled Java, $\mathrm{C}++$, and native/library code. Interpreted Java methods are only labelled as Interpreter, and cannot be identified with perf/perf-map-agent. However, call-stacks and flamegraphs produced with async-profiler can identify methods that undergo interpreted execution. It is a fairly trivial matter, using options for async-profiler and perf to generate flamegraphs where thread PIDs are also captured with callstacks, this can sometimes be beneficial, but it can quickly become confusing if an application contains many threads.

Identifying Inlined Methods. In Figure 1, an inlined method appears as a teal/blue frame above a green Java JIT compiled method, further inlined methods appear as stacks of teal/blue frames. Inlining information accuracy is improved through the use of $-\mathrm{XX}:+$ DebugNonSafePoints. Note that flamegraph columns where a teal/blue rectangle/method is directly below green, indicate a place where the

\footnotetext{
${ }^{1}$ Skid occurs when the reported program counter $(\mathrm{PC})$ value is a number of instructions away from the actual PC when the thread was interrupted for stack sampling.
}

JIT compiled and inlined code has called a method that was chosen not to be inlined. Note that on-core flamegraphs, only demonstrate stacks for threads that were executing when sampling of call stacks occurred. They neglect the importance of off-core time when threads are blocked.

Heap/Allocation Flamegraphs. Figure 2 is an heap allocation flamegraph created using async-profiler, here the width of a call stack is proportional to the amount of heap memory allocation. The topmost call-stack element represents the allocated data type, i.e. an array, such as char [], or an object such as StandardFilter. The profiler features TLAB-driven sampling that relies on callbacks (see AllocTracer in the OpenJDK/HotSpot sources) to receive notifications; i), when an object is allocated inside a newly created TLAB (annotated as INSIDE or aqua in Figure 2), and ii), when an object is allocated on a slow path outside a TLAB (annotated as OUTSIDE or in orange in Figure 2), for example when its size exceeds that of the TLAB. This means not every allocation is counted, but only allocations every $\mathrm{N} \mathrm{kB}$, where $\mathrm{N}$ is the average size of TLAB. This makes heap sampling very cheap and suitable for production, as in practice it will often reflect the top allocation sources, but the collected data may be incomplete.

Offwaketime Flamegraphs. The blocking latency associated with off-cpu time can be analysed with offwaketime flamegraphs. Figure 3 presents cropped illustrative output of the BCC/eBPF tool offwaketime for the avrora benchmark for blocking of thread node- 0 . The bottom stacks, colored blue, are off-core stacks that indicate the sequence of method calls leading to a thread blocking, and these are read from the bottom up leading towards the point where they blocked, that is separated from the waking thread stack by a grey block that is labelled --. The waking thread stacks, colored aqua, have their call-stack frames listed in reverse order, and are read from the top down, from application code, down to the method that caused the blocked thread's scheduling state to change. The tool of fwaketime achieves similar information to [14] for determining the blocking and wakeup behavior without requiring a modified JVM. A current limitation is that one wakeup stack may not be sufficient to explain all the sources of blocking latency. For example, when the "waker-thread" was itself blocked on another thread, potentially leading to a chain of blocking and wakeup stacks.

\section{JAVA BASED PROFILING}

Sampling profilers repeatedly collect snapshots of the call-stack of functions/methods, that describe the on-core execution context of a thread running on a processing core, at the sampled moments in time. The thread execution time of specific methods is statistically related to the number of times the method appears in all stack traces - as long as the samples are collected fairly, and that all points in a program run are selected randomly to remain statistically independent. If many samples are collected over time, and the code stack-traces are highly correlated, then we can safely assume that threads in a program are spending most of their time executing code described by the most heavily correlated call-stack. Unfortunately, profilers that directly rely on the fava Virtual Machine Tool 


\begin{tabular}{|c|c|c|c|c|}
\hline Tool & async-profiler & perf & bcc-java & offwaketime \\
\hline Native applications & No & $\begin{array}{c}\text { Yes } \\
\end{array}$ & $\begin{array}{c}\text { Yes } \\
\end{array}$ & $\begin{array}{c}\text { Yes } \\
\end{array}$ \\
\hline JIT compiled code support & Automatic & requires perf-map-agent & Not appropriate & requires perf-map-agent \\
\hline JVM Restrictions & $\begin{array}{c}\text { OpenJDK/HotSpot } \\
\text { AsyncGetCallTrace } \\
\text { TLAB overflow callbacks } \\
\text { JDK } 7 \mathrm{u} 40\end{array}$ & $\begin{array}{c}\text { JDK 8u60 } \\
\text { onwards }\end{array}$ & $\begin{array}{l}\text { No, but for thread } \\
\text { names JDK9 needed }\end{array}$ & $\begin{array}{l}\text { JDK8u60 } \\
\text { onwards }\end{array}$ \\
\hline Interpreted methods & Identified & Seen as Interpreter & Not appropriate & Seen as Interpreter \\
\hline Identify inlined Java methods & No & $\begin{array}{c}\text { Yes } \\
\text {-XX:+DebugNonSafePoints }\end{array}$ & No & No \\
\hline $\begin{array}{l}\text { JDK -XX Flags } \\
\text { +PreserveFramePointer } \\
\text { +DebugNonSafePoints }\end{array}$ & $\begin{array}{l}\text { None } \\
\text { No } \\
\text { No }\end{array}$ & $\begin{array}{c}\text { Yes } \\
\text { Required } \\
\text { Improves inlining accuracy }\end{array}$ & $\begin{array}{l}\text { None } \\
\text { No } \\
\text { No }\end{array}$ & $\begin{array}{l}\text { Yes } \\
\text { Required } \\
\text { No }\end{array}$ \\
\hline Methodology & $\begin{array}{l}\text { Sampled } \\
\text { call-stack }\end{array}$ & $\begin{array}{c}\text { Sampled } \\
\text { call-stack }\end{array}$ & $\begin{array}{l}\text { eBPF tracing } \\
\text { instrumentation }\end{array}$ & $\begin{array}{l}\text { eBPF tracing blocked time } \\
\text { captures call-stacks }\end{array}$ \\
\hline PC stack sampling skid & Yes & Yes & No & No \\
\hline Heap profiling/overhead & TLAB based, low overhead & None & None & None \\
\hline Identify hot-methods & $\begin{array}{l}\text { Some JVM stubs/intrinsics } \\
\text { are invisible }\end{array}$ & $\begin{array}{l}\text { Full-stack, stubs \& } \\
\text { intrinsics are visible }\end{array}$ & Thread-based (No) & Blocked-time (No) \\
\hline Microarchitecture counters & Sample call-stacks & Sample call-stacks & $\begin{array}{c}\text { Measures changes } \\
\text { associated with threads }\end{array}$ & Measures off-core time \\
\hline Flamegraph visualisation & Yes & Yes & No & Yes \\
\hline $\begin{array}{l}\text { Relate blocked thread call-stacks } \\
\text { to wakeup call-stacks }\end{array}$ & No & No & No & $\begin{array}{l}\text { Yes - with selective } \\
\text { filtering on blocking time }\end{array}$ \\
\hline
\end{tabular}

Table 1: Summary of features and functionality of profiling and tracing tools used in experiments.

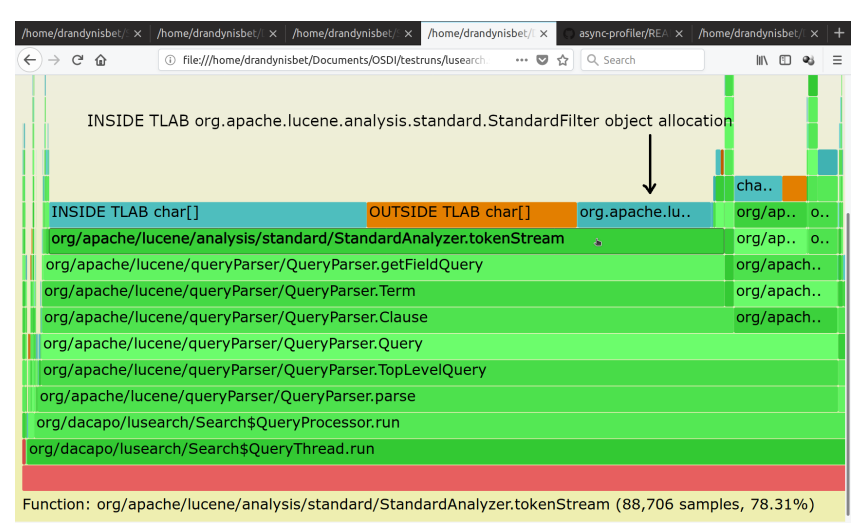

Figure 2: Annotated flamegraph, async-profiler for lusearch benchmark for heap memory allocations.

Interface (JVMTI) suffer from the problem of safe-point ${ }^{2}$ bias [18] The sampled data is skewed towards the times when threads have voluntarily yielded the processing core at safe-points where the JVTMI's GetStackTrace can directly obtain call-stacks. One might incorrectly come to the conclusion that the actual hot code should lie somewhere between the sampled call stack of the "hot" safe-point and the potential call-stack of the immediately previous safe-point Yet, this is not the case because there are significant, variable and lengthy delays between when an JVMTI agent signals an JVM to

\footnotetext{
${ }^{2}$ The JVM inserts safe-points that define points where the state of an executing thread is well understood. Effectively this means that all object references stored on the stack are at known locations, and actual storage locations can change during an GC.
}

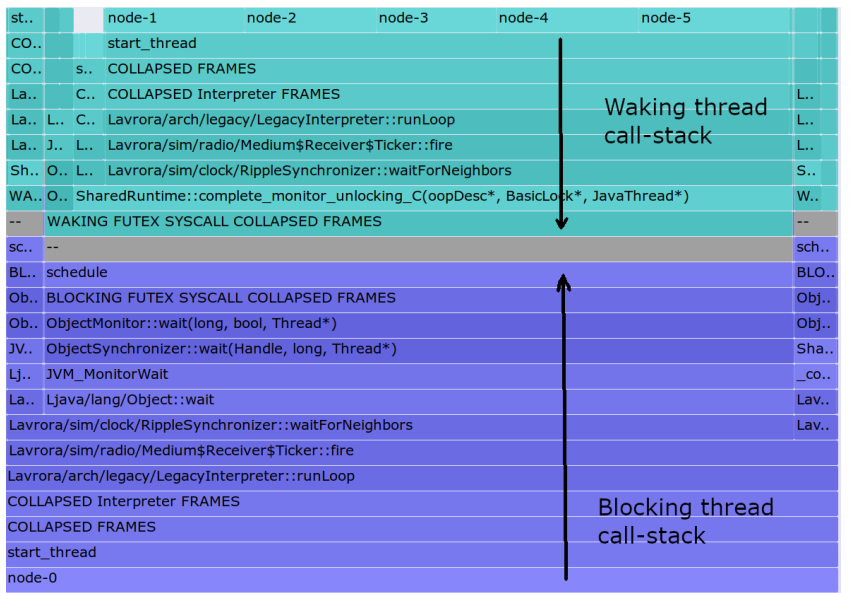

Figure 3: Cropped, annotated offwaketime flamegraph (restricted to thread node-0 blocking) for avrora benchmark.

sample a stack trace and the operation occurring. The JVMTI request is first added to the VM thread's work queue, and the VM thread must process any prior pending tasks first. Figure 1 demonstrates significant VM_Thread activity ( $20 \%$ of call stacks) related to GC.

Mytkowicz et. al. demonstrated, in [18] that four different commercial and open-source profilers often produced demonstrably different results for the percentage overhead of, and the actual hottest method. The overhead of profiling varied between $1.1 \mathrm{x}$ and $1.5 \mathrm{x}$ for the different profilers on sequential benchmarks chosen from $\mathrm{DaCapo}$, and different profilers caused significant perturbation 


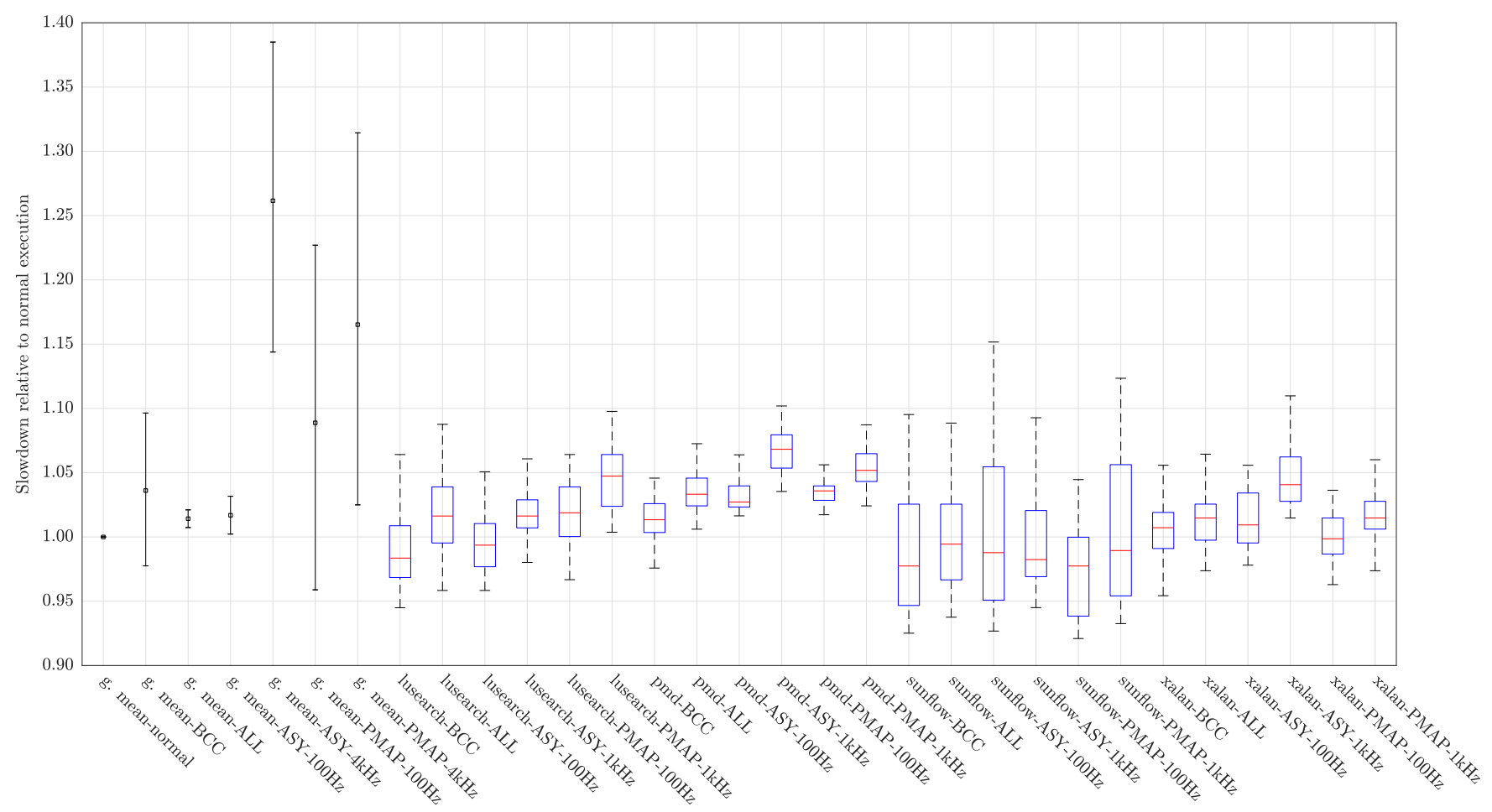

Figure 4: Normalized execution time overheads for unprofiled (normal) bcc-java (BCC), async-profiler on-core with (ASY) and heap allocation with (ALL), perf (PMAP) summarised for lusearch, pmd, sunflow, xalan benchmarks. g.mean refer to all 11 benchmarks studied

to the placement of safe-points in compiled methods. Safe-point placement is an implementation decision of the JVM, therefore different JVM's are likely to report hot-methods with different results and accuracy. Current popular and widely used profilers such as VisualVM, JProfile and YourKit all provide a sampling processingcore profiler that suffers from the problem of safe-point bias. Further problems concerning sampling profilers, and also for bytecode based instrumentation using JVMTI, is that any injected bytecode:-Introduces overhead to actually perform the stack trace collection. -Affects the accuracy of analysis and the applied JIT optimizations; Inlining decisions change if a method becomes too large to inline. GC and memory behavior in the JVM may be significantly altered if escape analysis [8] cannot decide if it is safe to allocate specific objects on the stack, and instead they are allocated on the heap.

-Changes in code size are likely to affect compiled code layout, and cache performance due to the need to respect machine alignment for specific data types.

-Will change safe-point placement because of injected bytecode.

A prototype safe-point bias free proof-of-concept profiler was first demonstrated in [18]. UNIX signals were used to pause Java application threads prior to sampling the call-stack of the currently executing method. A JVMTI agent was used to build a map of x86 code ranges to Java methods that enables code addresses in call-stacks to be mapped back to Java methods. Note, the profiler executes completely outside of the JVM, and cannot know the identity of an interpreted method.
The honest-profiler [3] and async-profiler [2,19] are recent examples that have extended and improved the proof of concept ideas of [18]. We do not discuss the honest-profiler due to space limitations. The async-profiler employs a hybrid approach for its stack trace collection based on i), AsyncGetCallTrace to obtain information on a thread's Java frames, without the requirement to be at a safe-point, and ii), perf to provide information on native code and kernel frames. The perf_event API is used to configure stack samples to be placed into a memory buffer, and a signal is delivered when a sample has been taken. Its signal handler then calls AsyncGetCallTrace to capture the Java stack that it then merges with the perf stack trace of native and kernel information.

The main benefits of this approach over a purely perf/eBPF approach is that it can be used to profile applications running on older JVM releases that do not support -XX:+PreserveFramepointer ${ }^{3}$ that is necessary for OS tool based stack walking of Java thread stacks. The main pros and cons of async-profiler are:

- AsyncGetCallTrace is an internal Oracle/OpenJDK API that is not guaranteed to be available in other JVM implementations.

- Optimised compiler inserted stub code that enables efficient implementation of compiler intrinsics, and performance critical JVM operations such as crc32 and System. arraycopy will generally be invisible to AsyncGetCallTrace because the JVM does not record the necessary metadata for many such stubs.

\footnotetext{
${ }^{3}$ Execution overhead of this flag was less than $4 \%$ in our experiments.
} 
- Off-core thread stacks that are blocked or sleeping are not collected by AsyncGetCallTrace.

- AsyncGetCallTrace will produce some failed stack traces where it is unable to safely walk a stack without causing an invalid memory access. There were very few failed stack traces in our experiments. perf also suffers from this unavoidable problem.

- Does not require generating a map file to map Java code addresses to method names, if one uses perf_event directly then one traditionally uses perf-map-agent for this purpose.

- Identifies the methods in interpreter frames, perf-map-agent merely identifies that an interpreter frame is present.

- Allocation profiling can be performed to identify the sites where the largest amount of heap memory is allocated. In our experiments the overhead of allocation profiling is always below $2.8 \%$. Allocation profiling does not impact on escape analysis or JIT optimizations.

The async-profiler tool can collect stack traces from Java methods, native calls, JVM code and kernel functions (only possible in processing core profiling mode). Both async-profiler and perf approaches support reading performance counters supported by perf_events such as:- last-level cache/data TLB load misses, L1-dcache misses, branch misses, page faults, and instructions retired. The perf approach collects callstacks and has access to perf_events, and uses the perf-map-agent JVMTI agent to generate a mapping of code addresses to Java symbol names. The main benefits of the perf-based approach over async-profiler are that: (1) the JVM flag $-X X$ : +DebugNonSafepoints enables inlining decisions to be accurately visualized in a flamegraph, and that (2) JVM generated stubs, and performance critical operations (e.g. JVM intrinsics), can be observed that are invisible to async-profiler.

No commercial features are required by async-profiler, as it is completely based on open-source technologies and works with OpenJDK from version $7 \mathrm{u} 40$ onwards, where the TLAB callbacks appeared. Note, the heap allocation functionality of async-profiler requires libjvm.so JVM debug symbols.

Stack Fragment Sampling (SFS). SFS, as described in [15], has less overhead than AsyncGetCallTrace and JVMTI based methods, this is achieved using a modified HotSpot JVM. SFS typically occurs only when a thread is active and on-core. A thread is paused to copy a fragment of its full stack to a buffer. Copied fragments are asynchronously retrieved from the buffer and decoded to full stack traces. Redundant samples are not captured giving reduced storage overheads.

Their work is of low overhead, but they encounter significant problems when enabling the collection of information on waiting periods, i.e. when a thread is blocked for I/O or to acquire a lock. The number of lost samples increases from a maximum of $2 \%$ for pmd, to more than $50 \%$ for the benchmarks actors, avrora, and scalatest in their experiments. Further, SFS operational overheads are likely to be lower on their quad-core experimental setup in comparison to our 8 core node. The performance slowdown of their techniques was approximately $1.15 x$ (i.e. $15 \%$ slower) for avrora when waiting time was not sampled for a $10 \mathrm{kHz}$ sampling rate. They found i) that their heuristic rules to enable stack walking work for more than $90 \%$ of sampled stacks, even when execution is in native or VM code, and ii), that a fragment size of $32 \mathrm{~KB}$ can obtain complete stack traces.

\section{THE BCC-JAVA TOOL}

The Berkeley Packet Filter (BPF) [22] enabled instrumentation code to be attached to a network socket by injecting bytecode (describing an BPF program) from userspace to the kernel. JIT compilation and verification checks are performed to ensure no security or kernel crashes can occur. The development of extended BPF (eBPF) has (i) improved JIT compilation, (ii) added many more probes to Linux, and (iii) enabled user-level statically defined trace probe points for application programs. Probes are points where dynamic instrumentation can be attached. Each probe has a specific set of variables that are used to describe the probe's events. The instrumented code can perform different processing based on the values of the variables of a probe.

We use the BCC toolkit that provides a set of Python scripting examples demonstrating how to; i), launch BCC tools, ii), exploit eBPF features for instrumentation, and iii), how to efficiently perform $\mathrm{I} / \mathrm{O}$ to obtain profiling/tracing results.

The main capabilities of BCC, and eBPF are summarized in [12]. Instrumented code can read hardware performance counters, and update arbitrary data structures to collect and process information. A Maps data abstraction enables information to be processed and aggregated in kernel, before sharing with user-space when required.

We have developed a low-overhead tracing tool that records microarchitectural and execution time characteristics associated with the multi-threaded execution of Java programs. The tool currently records a thread's name, its start/exit time, cycles, instructions, and time spent on-core in execution. We additionally record the number of scheduling quanta given to each thread, the off-core waiting time, and the total number of sys_futex system calls. The initial JVM process is created by executing the JDK java command; hereafter referred to as the java-process. Our tool uses instrumentation of: - java-process creation and termination by instrumenting kernel methods for sched_process_exec, sched_process_fork and sched_process_exit. The PID of the initial java-process that is created is stored in a hashmap.

- The creation of new threads that are children of the initial PID are tracked using a kernel probe attached to the sys_clone method. - Kernel probes are attached to the entry and exit of the sys_futex system call. We record for each thread, the number of futex calls, the total elapsed time whilst blocked, and performance counter values when a thread is blocked/unblocked.

- Thread scheduling, namely sched_switch is instrumented to collect time and performance counter information concerning the allocation of a thread to a processing core. The counter values and elapsed time are updated at the end of a thread's quantum, or when the thread enters the sys_futex system call.

- On termination of the java-process, the tool stores per-thread details of accumulated performance counter values, total wait time, total calls to sys_futex, and elapsed time. OpenJDK9 uses a default thread naming scheme that allows to distinguish between different types of threads, i.e. application and service threads related to JIT compilation, GC, and other JVM services. Thus, measurements are linked to specific JVM subsystems. 


\section{EXPERIMENTS - TOOLS OVERHEADS}

The experimental objective is to quantify the execution time overheads of perf, async-profiler and bcc-java tools using the established set of DaCapo [5] JVM performance evaluation benchmarks. The overheads of running the different performance tools are normalized against the execution times of ELEVEN unmodified (avrora, fop, h2, jython, luindex, lusearch, pmd, sunflow, tradebeans, tradesoap, xalan) DaCapo-bach- 9.12 benchmarks that execute successfully under OpenJDK9 with G1 GC using default application problem and heap sizes.

The experimental machine is a two-socket Intel Xeon E5-2690, with 8 physical cores per socket (hyper-threading is disabled), 20MB LLC cache, and a total of 384GB DRAM main memory, with Linux kernel 4.13.0-36-generic. All cores are set to $2.9 \mathrm{GHz}$ frequency via a user-space governor. No other users are present on the system, a single ssh connection is used to initiate and monitor experiments that only access local disks. NUMA memory effects are avoided by executing applications only on cores belonging to one socket, as in [21]. We used bcc-tools version 0.5.0-1, and OpenJDK (build 9-internal+0-2016-04-14-19524-6.buildd.src, mixed mode).

Each benchmark is executed for 20 iterations and the 19th iteration's time is recorded. This is repeated 30 times in order to avoid variability due to JIT recompilation and startup costs. The 19th iteration execution time measurements are normalized to the average of each benchmark under normal execution without profiling over its 30 runs. Figure 4 depicts the median execution times, in the form of box plots, normalized to average execution time without sampling. On each box, the central mark denotes the median, and the bottom and top edges indicate the 25 th and 75 th percentiles, respectively. The box spans the interquartile range of the sampled data. The whiskers extend to the most extreme data points not considered outliers, which are not shown. However, the whiskers extend to up to $1.5 \mathrm{x}$ of the interquartile range below and above the bottom and top edges, respectively. This corresponds to approximately $\pm 2.7 \sigma$ or 99.3\% coverage if the data were normally distributed. The g. mean labels in Figure 4 show the geometric means over all ELEVEN benchmarks; e.g., g. mean-ASY $-100 \mathrm{~Hz}$ is the geometric mean over all benchmarks of async-profiler on-core profiling with $100 \mathrm{~Hz}$ sampling. These are accompanied by error bars that delimit $95 \%$ confidence intervals.

The experimental configurations are: (1) baseline (no profiling tools used), (2) async-profiler with ALL allocation and ASY processing core profiling, (3) perf with perf-map-agent (notated as PMAP), and (4) bcc-java based tracing (notated as BCC) where we measure the instructions and cycles executed along with accumulated counts of execution/waiting time and the start and end times of individual threads. For on-core async-profiler and perf we used $100 \mathrm{~Hz}, 1 \mathrm{kHz}$ and $4 \mathrm{kHz}$ sampling frequencies. Note, $10 \mathrm{kHz}$ perf sampling was not feasible as kernel warning messages appeared concerning interrupts and frequency throttling.

For clarity, Figure 4 only plots sampling frequencies of $100 \mathrm{~Hz}$ and $1 \mathrm{KHz}$ for a small set of individual benchmarks (lusearch, pmd, sunflow and xalan), whilst geomean plots refer to execution of all ELEVEN benchmarks with $100 \mathrm{~Hz}$ and $4 \mathrm{KHz}$ sampling, in this way we can see the relatively higher overheads of async-profiler (1.26x ASY-4kHz) in comparison to perf $(1.16 \mathrm{x}$ PMAP-4kHz) at higher sampling rates (perf overhead is substantially less than async-profiler for $4 \mathrm{kHz}$ sample rates for all benchmarks other than avrora). This is despite the unusually high overhead of perf on avrora that is more than $2 x$ even at a low $100 \mathrm{~Hz}$. However on a laptop class machine with an i7-7500U processor having 2 physical processing cores (4-hyperthreaded) @2.7GHz, the overhead of the same experiment was $3.8 \%$. We cannot definitively explain why there is a significant overhead even for $100 \mathrm{~Hz}$ sampling, but we suspect that processor chipset issues, and kernel version issues such as default perf buffer sizes may be relevant.

Our bcc-java tool has very low-overhead apart from avrora where its average overhead is $1.37 \mathrm{x}$. bcc-java text output enabled us to determine that the Java application threads were spending circa $80 \%$ of their total execution time blocked off-core, largely because of repeated calls to sys_futex when attempting to acquire locks. bcc-java has relatively high overhead on avrora because instrumentation measuring time, cycles and instructions executed, along with the code to update the in-kernel eBPF maps, occurs with relatively high frequency, that we calculated from bcc-java tracing to occur on average every 9863 cycles and 12658 instructions executed, by examining bcc-java tracing information for one iteration of one application thread on avrora.

\section{PERFORMANCE USE-CASE GUIDELINES}

The next paragraphs present use-cases and the suggested guidelines for how to identify them.

A) Identifying the call-stack context, load-imbalance, and functions where significant execution time is spent. Browser views of on-core flamegraphs produced via perf, or using async-profiler are appropriate. The method with the most significant execution time equates to finding the top-most widest call-stack tower. Note that some functions may appear in many different call-stacks, because they are called from many different call-sites, and this may cumulatively add up to be a significant proportion of the total number of stack-samples, and hence execution time. Searching for a function name in the browser view of an SVG will determine the total \% appearances of the function in all call-stack samples. perf can see many JVM intrinsics, compiler stubs and inlining decisions that are mostly invisible to async-profiler. Load-imbalance can be identified by using flamegraphs that propagate thread PIDs to the call-stack. Unequal width call-stacks concerning different thread PIDs will signify unequal on-core load-imbalance across threads. Load-imbalance can also measured by examining thread start/stop and blocked execution times that is traced by the bcc-java tool.

B) Identifying if GC time, and memory allocation issues are significant Core-profiled flamegraphs can be used to identify if GC related methods are significant, whereas allocation based flamegraphs with async-profiler can determine the source code methods where significant memory allocation occurs, and whether allocations were from inside or outside of a TLAB. Manually restructuring the code to reduce the volume of memory allocated, and applying standard GC log file analysis tools to change and optimize specific GC algorithm parameters are recommended.

C) Identifying FIT compilation method inlining decisions that may be critical for performance. We recommend, using perf, with $-X X$ :+DebugNonSafePoints enabled. Flamegraph columns where 
a teal/blue rectangle is placed directly below a green rectangle indicates a call site where JIT compiled and inlined code has called a method that was chosen not to be inlined. JIT compilation logging can be selectively enabled for the affected methods. A tool such as JITWatch [1] may help to determine the reasons why inlining failed. Careful refactoring of large methods into multiple smaller ones may enable inlining decisions that were previously precluded.

D) Identifying the causes of blocked thread execution states, for example, lock-contention, I/O, and a lack of processing resources. The bcc-java tool can be used to identify on a per-thread basis if a thread spends a significant amount of time blocked, and, also if it relates to sys_futex lock acquisition/release. The eBPF/BCC tool offwaketime can be used to identify the specific call-stacks that contribute to long blocking times, and also the call stacks that cause the blocked threads to be woken up in the kernel. This achieves similar functionality to [14] but without any JVM modifications. Note, the of fwaketime tool uses tracing so the actual blocked execution time is measured. Command line arguments can be used to filter out very short and extremely long thread blocking times such as when a server is lightly loaded or inactive.

\section{CONCLUSIONS}

This paper examines the peformance analysis capabilities of asyncprofiler, perf/perf-map-agent and the potential of eBPF-based tools such as our bcc-java, and offwaketime. Flamegraph visualizations of memory allocation (GC) and processing core profiling can easily show which Java methods and native or OS code are significant, and that even JIT compilation inlining decisions can be captured. Sampling at $1 \mathrm{kHz}$ (1000 samples per second) offers reasonable overhead and $100 \mathrm{~Hz}$ sampling rates are suitable for production use, except for avrora with perf. Although not presented in the paper, the async-profiler and the perf/perf-map-agent approaches can both be used to attribute microarchitecture performance counter events to flamegraphs. However, PC sampling skid (affecting both async-profiler and the perf/perf-map-agent) will vary depending on the specific performance counter event and processor implementation. We have found that sampling-based profiler flamegraph visualizations provide a useful tool to investigate and to characterize Java application and GC performance. In contrast, our tool bcc-java does not suffer from the PC-sampling skid problem as it is a tracing based tool. The overhead of bcc-java is small (geomean 3.6\%) despite capturing microarchitectural performance counters on thread scheduling activities.

\section{REFERENCES}

[1] AdoptOpenJDK. 2018. Adoptopenjdk jitwatch home page. https://github.com/ AdoptOpenJDK/jitwatch. (2018).

[2] Andre Pangin et al. 2018. Async-profiler. https://github.com/jvm-profilingtools/async-profiler,changeset=0a6f365. (2018).

[3] Richard Warburton et al. 2018. Honest profiler git repository. https://github. com/jvm-profiling-tools/honest-profiler. (2018).

[4] Bowen Alpern et al. 1999. Implementing jalapeño in Java. In Proceedings of the 14th ACM SIGPLAN Conference on Object-oriented Programming, Systems, Languages, and Applications (OOPSLA '99). ACM, Denver, Colorado, USA, 314324. ISBN: 1-58113-238-7. DOI: 10.1145/320384.320418. http://doi.acm.org/10. $1145 / 320384.320418$

[5] Stephen M. Blackburn et al. 2006. The DaCapo benchmarks: Java benchmarking development and analysis. In Proceedings of the 21st Annual ACM SIGPLAN Conference on Object-oriented Programming Systems, Languages, and Applications (OOPSLA '06). ACM, Portland, Oregon, USA, 169-190. ISBN: 1-59593-348-4. DOI: $10.1145 / 1167473.1167488$. http://doi.acm.org/10.1145/1167473.1167488.
[6] Ting Cao, Stephen M Blackburn, Tiejun Gao, and Kathryn S McKinley. 2012. The yin and yang of power and performance for asymmetric hardware and managed software. In Proceedings of the 39th Annual International Symposium on Computer Architecture (ISCA '12). IEEE Computer Society, Portland, Oregon, 225-236. ISBN: 978-1-4503-1642-2. http://dl.acm.org/citation.cfm?id=2337159. 2337185.

[7] Dehao Chen, Neil Vachharajani, Robert Hundt, Shih-wei Liao, Vinodha Ramasamy, Paul Yuan, Wenguang Chen, and Weimin Zheng. 2010. Taming hardware event samples for fdo compilation. In Proceedings of the 8th Annual IEEE/ACM International Symposium on Code Generation and Optimization (CGO '10). ACM, Toronto, Ontario, Canada, 42-52. ISBN: 978-1-60558-635-9. DoI: 10.1145/1772954.1772963. http://doi.acm.org.manchester.idm.oclc.org/10.1145/ 1772954.1772963

[8] Jong-Deok Choi, Manish Gupta, Mauricio Serrano, Vugranam C. Sreedhar, and Sam Midkiff. 1999. Escape analysis for Java. In Proceedings of the 14th ACM SIGPLAN Conference on Object-oriented Programming, Systems, Languages, and Applications (OOPSLA '99). ACM, Denver, Colorado, USA, 1-19. ISBN: 1-58113238-7. DOI: 10.1145/320384.320386. http://doi.acm.org/10.1145/320384.320386.

[9] Oracle Corporation. 2013. Advanced Java diagnostics and monitoring without performance overhead. (2013). http://www.oracle.com/technetwork/java/ javaseproducts/mission-control/java-mission-control-wp-2008279.pdf.

[10] GCeasy. 2018. Gc easy universal gc log analyzer. Web Page. (2018). http:// gceasy.io/.

[11] GCViewer. 2017. Open source fork of tagtraum industries gcviewer. https: //github.com/chewiebug/GCViewer. (2017).

[12] Brendan Gregg. 2018. Linux enhanced bpf (ebpf) tracing tools. (2018). Retrieved from http://www.brendangregg.com/ebpf.html.

[13] Brendan Gregg. 2016. The flame graph. Queue, 14, 2, Article 10, (Mar. 2016), 10:91-10:110. ISSN: 1542-7730. DOI: 10.1145/2927299.2927301. http://doi.acm. org/10.1145/2927299.2927301.

[14] Peter Hofer, David Gnedt, Andreas Schörgenhumer, and Hanspeter Mössenböck. 2016. Efficient tracing and versatile analysis of lock contention in Java applications on the virtual machine level. In Proceedings of the 7th ACM/SPEC on International Conference on Performance Engineering (ICPE '16). ACM, Delft, The Netherlands, 263-274. ISBN: 978-1-4503-4080-9. DOI: 10.1145/2851553.2851559. http://doi.acm.org/10.1145/2851553.2851559.

[15] Peter Hofer and Hanspeter Mössenböck. 2014. Fast Java profiling with schedulingaware stack fragment sampling and asynchronous analysis. In Proceedings of the 2014 International Conference on Principles and Practices of Programming on the fava Platform: Virtual Machines, Languages, and Tools (PPPJ '14). ACM, Cracow, Poland, 145-156. ISBN: 978-1-4503-2926-2. DoI: 10.1145/2647508.2647509. http://doi.acm.org/10.1145/2647508.2647509.

[16] Philipp Lengauer, Verena Bitto, and Hanspeter Mössenböck. 2015. Accurate and efficient object tracing for Java applications. In Proceedings of the 6th ACM/SPEC International Conference on Performance Engineering (ICPE '15). ACM, Austin, Texas, USA, 51-62. ISBN: 978-1-4503-3248-4. DOI: 10.1145/2668930.2688037. http://doi.acm.org/10.1145/2668930.2688037.

[17] Philipp Lengauer, Verena Bitto, Hanspeter Mössenböck, and Markus Weninger. 2017. A comprehensive Java benchmark study on memory and garbage collection behavior of DaCapo, DaCapo Scala, and SPECjvm2008. In Proceedings of the 8th ACM/SPEC on International Conference on Performance Engineering (ICPE '17). ACM, L'Aquila, Italy, 3-14. ISBN: 978-1-4503-4404-3. DOI: 10.1145/3030207.3030211. http://doi.acm.org/10.1145/3030207.3030211.

[18] Todd Mytkowicz, Amer Diwan, Matthias Hauswirth, and Peter F. Sweeney. 2010. Evaluating the accuracy of Java profilers. In Proceedings of the 31st ACM SIGPLAN Conference on Programming Language Design and Implementation (PLDI '10). ACM, Toronto, Ontario, Canada, 187-197. ISBN: 978-1-4503-0019-3. DOI: 10.1145/1806596.1806618. http://doi.acm.org/10.1145/1806596.1806618.

[19] Andre Pangin and Vadim Tsesko. 2017. The art of jvm profiling. JPoint International Developer Conference. (2017). http://2017.jpoint.ru/en/talks/the-art- ofjvm-profiling/.

[20] Git Project. 2018. Visualvm home page. https://visualvm.github.io/. (2018).

[21] J.B. Sartor, K.D. Bois, S. Eyerman, and L. Eeckhout. 2017. Analyzing the scalability of managed language applications with speedup stacks. In Proceedings of the IEEE Int. Symp. Performance Analysis of Systems and Software (ISPASS), 23-32.

[22] Valentine Sinitsyn. 2014. Berkeley packet filters with scapy (and friends). Linux f., 2014, 242, Article 3, (June 2014). IssN: 1075-3583. http://dl.acm.org/citation. cfm?id=2642922.2642925.

[23] Cheng-Chun Tu, Joe Stringer, and Justin Pettit. 2017. Building an extensible open vswitch datapath. SIGOPS Oper. Syst. Rev., 51, 1, (Sept. 2017), 72-77. ISSN: 0163-5980. DoI: 10.1145/3139645.3139657. http://doi.acm.org/10.1145/3139645. 3139657.

[24] YourKit. 2018. Object allocation recording. (2018). Retrieved from https://www. yourkit.com/docs/java/help/allocations.jsp. 